\title{
Pd single-atom monolithic catalyst: Functional 3D structure and unique chemical selectivity in hydrogenation reaction
}

\author{
Zedong Zhang ${ }^{1 \dagger}$, Min Zhou ${ }^{2 \dagger}$, Yuanjun Chen ${ }^{1 *}$, Shoujie Liu ${ }^{3}$, Haifeng Wang ${ }^{2 *}$, Jian Zhang ${ }^{1}$, \\ Shufang Ji ${ }^{1}$, Dingsheng Wang ${ }^{1 *}$ and Yadong $\mathrm{Li}^{1}$
}

\begin{abstract}
Regulating the selectivity of catalysts in selective hydrogenation reactions at the atomic level is highly desirable but remains a grand challenge. Here we report a simple and practical strategy to synthesize a monolithic single-atom catalyst (SAC) with isolated Pd atoms supported on bulk nitrogen-doped carbon foams (Pd-SAs/CNF). Moreover, we demonstrate that the single-atom Pd sites with unique electronic structure endow Pd-SAs/CNF with an isolated site effect, leading to excellent activity and selectivity in 4nitrophenylacetylene semi-hydrogenation reaction. In addition, benefiting from the great integrity and excellent mechanical strength, monolithic Pd-SAs/CNF catalyst is easy to separate from the reaction system for conducting the subsequent recycling. The cyclic test demonstrates the excellent reusability and stability of monolithic Pd-SAs/CNF catalyst. The discovery of isolated site effect provides a new approach to design highly selective catalysts. And the development of monolithic SACs provides new opportunities to advance the practical applications of single-atom catalysts.
\end{abstract}

Keywords: single-atom, monolithic catalyst, isolated site, hydrogenation reaction

\section{INTRODUCTION}

Regulating the selectivity of catalysts has become a significant research focus in catalysis [1-5]. Selective hydrogenation reactions are widely used in industrial manufacture of fine chemicals and pharmaceuticals [6-9]. In selective hydrogenation reactions, it remains elusive to selectively converting one specific functional group to obtain the desired product when other competitive functional groups locate at the same molecule. For instance, the reducible alkynyl and nitro functional groups are simultaneously hydrogenated by conventional nanocatalysts, resulting in a poor selectivity. Great progress has been made in tuning the selectivity by adjusting the interfacial electronic structure or lattice stress of nanocatalysts, which can alter the adsorption and activation properties of reactant species [10-15]. However, precise control of the structure of nanocatalysts is difficult at the atomic level; therefore, it poses a bottleneck to advancing atomic understanding of catalytic behaviors [16,17].

Featured with maximum atom-utilization efficiency and fully exposed active sites, single-atom catalysts (SACs) have attracted extensive attention and exhibited remarkable catalytic performance in various reactions [18-22]. The structural uniformity of SACs makes them serve as a model system to study the reaction mechanism at the atomic scale, which provides a great platform for atomic-level insight in regulating selectivity [23-25]. Moreover, spatial isolated sites and unique electronic structure, distinguishing from nanocatalysts, may endow SACs with minimizing choices and specific configuration of binding modes of substrates, which favors highly selective catalysis [26-31]. Currently, it is of great importance to reveal whether SACs can break through the dilemma of nanocatalysts in selective catalysis. It is worth mentioning that the previously reported SACs are in the form of powders, which limits their further practical applications.

\footnotetext{
${ }^{1}$ Department of Chemistry, Tsinghua University, Beijing 100084, China

${ }^{2}$ Key Laboratory for Advanced Materials, Centre for Computational Chemistry and Research Institute of Industrial Catalysis, East China University of Science and Technology, Shanghai 200237, China

${ }^{3}$ Chemistry and Chemical Engineering of Guangdong Laboratory, Shantou 515063, China

${ }^{\dagger}$ These authors contributed equally to this work.

* Corresponding authors (emials: chenyjfj@163.com (Chen Y); hfwang@ecust.edu.cn (Wang H); wangdingsheng@mail.tsinghua.edu.cn (Wang D))
} 
Hierarchical three-dimensionl (3D) carbon nanomaterials (HTCs) as the monolithic materials are widely used in the field of energy storage [32], oil-water separation [33,34] and sensor techniques [35]. However, studies about the design of HTCs as monolithic heterogeneous catalysts are limited. Actually, given the regular hierarchical pores and abundant defects in carbon lattices, HTC is a promising substrate to synthesize monolithic catalysts, which are more attractive in chemical industry because of their better mass transfer efficiency, no significant amplification effect, easier separation and regeneration compared with the conventional powder heterogeneous catalysts. Therefore, it is highly desirable to combine the merits of monolithic catalysts and SACs to develop monolithic SACs; however, it still remains a grand challenge.

Here, we report a facile and practical strategy to prepare monolithic SACs with isolated Pd single atoms anchored on carbon nitrogen foams (Pd-SAs/CNF) derived from the commercial assembled melamine foams (MFs). Furthermore, we demonstrate an isolated site effect to control the selectivity of $\mathrm{Pd}-\mathrm{SAs} / \mathrm{CNF}$ in hydrogenation reaction. Such isolated site effect makes Pd-SAs/CNF preferentially adsorb alkynyl group rather than the nitro group and inhibit further hydrogenation of alkenyl group owing to limited coordination space, which results in the catalytic production of 4-nitrostyrene with $\sim 99 \%$ conversion and $\sim 99 \%$ selectivity in 4-nitrophenylacetylene (NPA) semi-hydrogenation. Moreover, benefiting from great integrity and excellent mechanical strength, the monolithic Pd-SAs/CNF catalyst is easy to separate from reaction system for subsequent recycling.

\section{EXPERIMENTAL SECTION}

\section{Materials}

Melamine sponges $(99 \mathrm{~mm} \times 59 \mathrm{~mm} \times 20 \mathrm{~mm}$, Beijing Clean), palladium(II) acetylacetonate (99\%, Alfa), sodium (II) tetrachloropalladate (99\%, Alfa), commercial $\mathrm{Pd} / \mathrm{C}$ (20\%, Alfa), potassium iodide (99\%, Xilong chemical Co., Ltd.), polyvinylpyrrolidone (K23-27, Aladdin), formamide (99\%, Xilong chemical Co., Ltd.), NPA (97\%, Adamas), borane-ammoniacomplex (97\%, Shanghai 9dingchem Co., Ltd.) and methanol (99\%, Beijing Chemical Reagents, China) were used without further purification.

\section{Catalysts preparation}

Synthesis of Pd-SAs/CNF

The melamine sponges were firstly tailored to small pie- ces $(\sim 200 \mathrm{mg}, 2 \mathrm{~mm} \times 2 \mathrm{~mm} \times 2.5 \mathrm{~mm})$ and then washed with distilled water and methanol for three times, respectively. The obtained sponges were dried at $60^{\circ} \mathrm{C}$ under vacuum for $6 \mathrm{~h}$ and then soaked in the $30 \mathrm{~mL}$ methanol solution containing palladium(II) acetylacetonate $\left(2 \mathrm{mg}, \mathrm{Pd}(\mathrm{acac})_{2}\right)$. Then, the closed container containing the above mixture was placed on a shaker purchased from Kylin-Bell Lab Instruments. The shaker worked with $60 \mathrm{r} \mathrm{min}^{-1}$ for $6 \mathrm{~h}$ at room temperature to ensure that the sponges were fully wetted by the $\mathrm{Pd}(\mathrm{acac})_{2}$ solution. Subsequently, the as-prepared samples, marked as $\mathrm{Pd}^{2+} / \mathrm{MF}$, were washed with methanol to remove the $\operatorname{Pd}(\mathrm{acac})_{2}$ physically adsorbed on sponges. Then, the $\mathrm{Pd}^{2+} / \mathrm{MF}$ sample was squeezed, dried under vacuum at $60^{\circ} \mathrm{C}$ for $12 \mathrm{~h}$ and placed in a tube furnace and heated at $800^{\circ} \mathrm{C}$ for $30 \mathrm{~min}$ under flowing argon gas with the heating rate of $5^{\circ} \mathrm{C} \mathrm{min}^{-1}$. Finally, the Pd-SAs/CNF catalyst was prepared. The content of Pd species in Pd-SAs/ $\mathrm{CNF}$ was determined as $0.024 \mathrm{wt} \%$ by the inductively coupled plasma atomic emission spectrometry (ICP-AES) analysis.

\section{Synthesis of CNF and Pd-NPs/CNF}

The CNF sample was prepared with the same synthesis process of Pd-SAs/CNF except the addition of $\mathrm{Pd}(\mathrm{acac})_{2}$.

Pd nanoparticles (NPs) were firstly synthesized [36] for the synthesis of Pd-NPs/CNF. Poly(vinyl pyrrolidone) $(50 \mathrm{mg}, 0.45 \mathrm{mmol})$ and $\mathrm{KI}(17 \mathrm{mg}, 0.1 \mathrm{mmol})$ were dissolved into formamide $(5 \mathrm{~mL})$ and heated to $120^{\circ} \mathrm{C}$. Then, $29.5 \mathrm{mg} \mathrm{Na}{ }_{2} \mathrm{PdCl}_{4}$ powder was added into the above solution and maintained at $120^{\circ} \mathrm{C}$ for $10 \mathrm{~min}$. Finally, the Pd NPs were washed and prepared into $1 \mathrm{mg} \mathrm{mL}^{-1}$ of methanol dispersion. Subsequently, the Pd-NPs/CNF was prepared with the same synthesis process of Pd-SAs/CNF except that the palladium(II) acetylacetonate $(2 \mathrm{mg}$, $\operatorname{Pd}(\mathrm{acac})_{2}$ ) was replaced by $2 \mathrm{~mL}$ methanol dispersion of Pd NPs. The content of Pd in the Pd-NPs/CNF was $0.013 \mathrm{wt} \%$ by ICP-AES analysis.

\section{Synthesis of melamine-formaldehyde polymer and nitrogen-doped carbon powder (NC)}

Typically, $3 \mathrm{~g}$ melamine was added into the mixture of $14 \mathrm{~mL}$ distilled water and $13 \mathrm{~mL}$ formaldehyde with ultrasonic treatment for $5 \mathrm{~min}$ at room temperature [37]. Then, $2.5 \mathrm{~mL}$ sodium hydroxide solution $\left(0.1 \mathrm{~mol} \mathrm{~L}^{-1}\right)$ was slowly added into the above solution with vigorous stirring. Then, the solution was kept at $60^{\circ} \mathrm{C}$ for $2 \mathrm{~h}$. Subsequently, the obtained product, marked as melamine-formaldehyde polymer, was washed with distilled water for three times and dried at $60^{\circ} \mathrm{C}$ in vacuum for 
$12 \mathrm{~h}$. The melamine-formaldehyde polymer was placed in a tube furnace and heated at $800^{\circ} \mathrm{C}$ for $30 \mathrm{~min}$ under flowing argon gas with the heating rate of $5^{\circ} \mathrm{C} \mathrm{min}^{-1}$. Finally, the NC sample was obtained.

\section{Synthesis of $\mathrm{Pd} / \mathrm{NC}$}

$\mathrm{Pd} / \mathrm{NC}$ sample was prepared with the same synthesis process of Pd-SAs/CNF except melamine sponge was replaced by the as-obtained melamine-formaldehyde polymer powder. Specifically, $200 \mathrm{mg}$ as-obtained melamine-formaldehyde polymer powder was dispersed into $30 \mathrm{~mL}$ methanol solution of $\mathrm{Pd}(\mathrm{acac})_{2}(2 \mathrm{mg})$. After stirring for $6 \mathrm{~h}$, the precipitate was separated, washed with methanol for two times, dried at $60^{\circ} \mathrm{C}$ under vacuum for $12 \mathrm{~h}$ and then heated at $800^{\circ} \mathrm{C}$ for 30 min under argon gas with the heating rate of $5^{\circ} \mathrm{C} \mathrm{min}{ }^{-1}$. Finally, the product was obtained and marked as $\mathrm{Pd} / \mathrm{NC}$.

\section{Synthesis of $\mathrm{Pd}-\mathrm{SAs} / \mathrm{NC}$}

The as-obtained NC (200 mg) was dispersed into $30 \mathrm{~mL}$ methanol of $\operatorname{Pd}(\mathrm{acac})_{2}(2 \mathrm{mg})$. After stirring for $6 \mathrm{~h}$, the precipitate was separated by centrifugation, washed with methanol for two times and then dried at $60^{\circ} \mathrm{C}$ under vacuum for $12 \mathrm{~h}$. Subsequently, the as-obtained precipitate was placed in a tube furnace and heated at $150^{\circ} \mathrm{C}$ for $1 \mathrm{~h}$ under flowing argon gas with the heating rate of $5^{\circ} \mathrm{C} \min ^{-1}$. Finally, the Pd-SAs/NC sample was obtained.

\section{Catalytic evaluation}

In a typical catalytic reaction, NPA $(0.2 \mathrm{mmol})$, boraneammonia complex $(1.2 \mathrm{mmol})$, Pd catalysts (Pd-SAs/ CNF, Pd-NPs/CNF, Pd-SAs/NC, Pd/C, Pd(acac) $)_{2}$, respectively) with $0.01 \mathrm{~mol} \% \mathrm{Pd}, 4.9 \mathrm{~mL}$ ethanol and $100 \mu \mathrm{L}$ distill water were added into a $15-\mathrm{mL}$ pressure tube. The reactions proceeded at $50^{\circ} \mathrm{C}$ for $3 \mathrm{~h}$, respectively. When they were cooled to room temperature, the reaction mixtures were extracted with ethyl acetate, respectively. The conversions and selectivities of Pd catalysts for the hydrogenation reactions were determined by gas chromatography-mass spectrometry (GC-MS) with dodecane as the internal standard.

\section{Characterization}

Scanning electron microscopy (SEM) images were used to characterize the morphologies of Pd-SAs/CNF taken by SU-8010. Transmission electron microscopy (TEM) images of catalysts were collected on a Hitachi H-800 TEM. High-resolution TEM (HRTEM) images and element dispersive spectroscopy (EDS) images were recorded by a JEOL JEM-2100F with electron acceleration energy of $200 \mathrm{kV}$. High-angle annular dark field scanning TEM (HAADF-STEM) images were taken by JEOL $200 \mathrm{~F}$ TEM operated at $200 \mathrm{keV}$, which was equipped with a probe spherical aberration corrector. Powder X-ray diffraction (XRD) spectra were obtained by using Rigaku RU-200b X-ray diffractometer equipped with $\mathrm{Cu} \mathrm{Ka}$ radiation $(\lambda=1.5406 \AA$ ). X-ray photoelectron spectroscopy (XPS) spectra were collected with ULVAC PHI Quantera. The ICP-AES (Optima $7300 \mathrm{DV}$ ) was used to measure the concentrations of palladium in different catalysts. The GC-MS analysis was subjected to ISQ GC-MS with an electrical capture detector (ECD, ThermoTrace GC Ultra) using a capillary column (TR-5MS, Thermo Scientific; length $30 \mathrm{~m}$, inner diameter $0.25 \mathrm{~mm}$, film $0.25 \mu \mathrm{m}$ ) The $\mathrm{X}$-ray absorption find structure (XAFS) spectra were taken at BL14W1 station in Shanghai Synchrotron Radiation Facility (SSRF, operated at $3.5 \mathrm{GeV}$ with a maximum current of $250 \mathrm{~mA}, \mathrm{Pd}$ K-edge under fluorescence excitation mode). The XAFS data of Pd-SAs/CNF sample were collected at room temperature in fluorescence excitation mode using a Lytle detector and Ru filter. Pd film and $\mathrm{PdO}$ were used as references and measured in a transmission mode using ionization chamber.

\section{Computational method}

All the spin-polarized calculations were performed using the Perdew-Burke-Ernzerhof (PBE) function [38] within the generalized gradient approximation (GGA), implemented in the Vienna Ab-initio Simulation Package (VASP) [39,40] code. The project-augmented wave (PAW) method $[41,42]$ was used to represent the corevalence electron interaction, and the valence electronic states were expanded in plane wave basis sets with a cutoff energy of $450 \mathrm{eV}$. The van der Waals interactions were considered using the density functional dispersion (DFT)-D3 method [43]. To ensure enough vacuum space, a vacuum space of $15 \AA$ in the $z$ direction was used between the periodic images. A periodic graphene sheet $(14.82 \mathrm{~A} \times 17.06 \mathrm{~A} \times 15.35 \mathrm{~A})$ with $96 \mathrm{C}$ atoms was constructed, and a $k$-mesh of $2 \times 1 \times 1$ was employed for all structures. For Pd (111) metal catalyst, a $p(6 \times 4)$ supercell with three layers was constructed, and the bottom one layer was fixed at bulk truncated position. All the transition states (TSs) were searched by the constrained optimization scheme $[44,45]$ and the convergence threshold of forces was set to $0.05 \mathrm{eV} \mathrm{A}^{-1}$.

The adsorption energies are defined as follows: $E_{\text {ads }}=$ $E_{\text {(adsorbate/surface) }}-E_{\text {(adsorbate) }}-E_{\text {(surface) }}$, where $E_{\text {(adsorbate/surface) }}$, $E_{\text {(adsorbate) }}$ and $E_{\text {(surface) }}$ are the total energies of the adsorbate binding to surface, free adsorbate in gas phase 
and clean surface, respectively. The more negative the $E_{\text {ads }}$ value is, the more strongly the adsorbate binds on the surface. It is worth noting that all the adsorption energies of atomic $\mathrm{H}$ were calculated using $\mathrm{H}_{2}$ as the reference, considering that used hydrogen source (ammonia borane) can easily dehydrogenate and release $\mathrm{H}_{2}$. In addition, for the adsorption/desorption processes, the large entropy contributions of gaseous molecules $(T \Delta S)$, including the vibrational, rotational and translational entropies, must be considered to estimate the Gibbs free energy change $(\Delta G)$ at the temperature of $323 \mathrm{~K}$. The $\Delta G$ of adsorption/ desorption process was estimated according to $\Delta G=\Delta H$
- TAS. The reaction enthalpy $(\Delta H)$ was approximated with the total energy difference $(\Delta E)$ neglecting the small zero-point energy correction $(\triangle \mathrm{ZPE})$, heat capacity correction and $\Delta(p V)$ term [46].

\section{RESULTS AND DISCUSSION}

The synthetic procedure is illustrated in Fig. 1a. Firstly, porous MF was soaked in methanol solution containing $\mathrm{Pd}(\mathrm{acac})_{2}$. Subsequently, the MF containing Pd precursor was dried and marked with $\mathrm{Pd}^{2+} / \mathrm{MF}$, followed by pyrolysis to obtain the final monolithic Pd-SAs/CNF catalyst. What's more, monolithic Pd-SAs/CNF catalyst can be
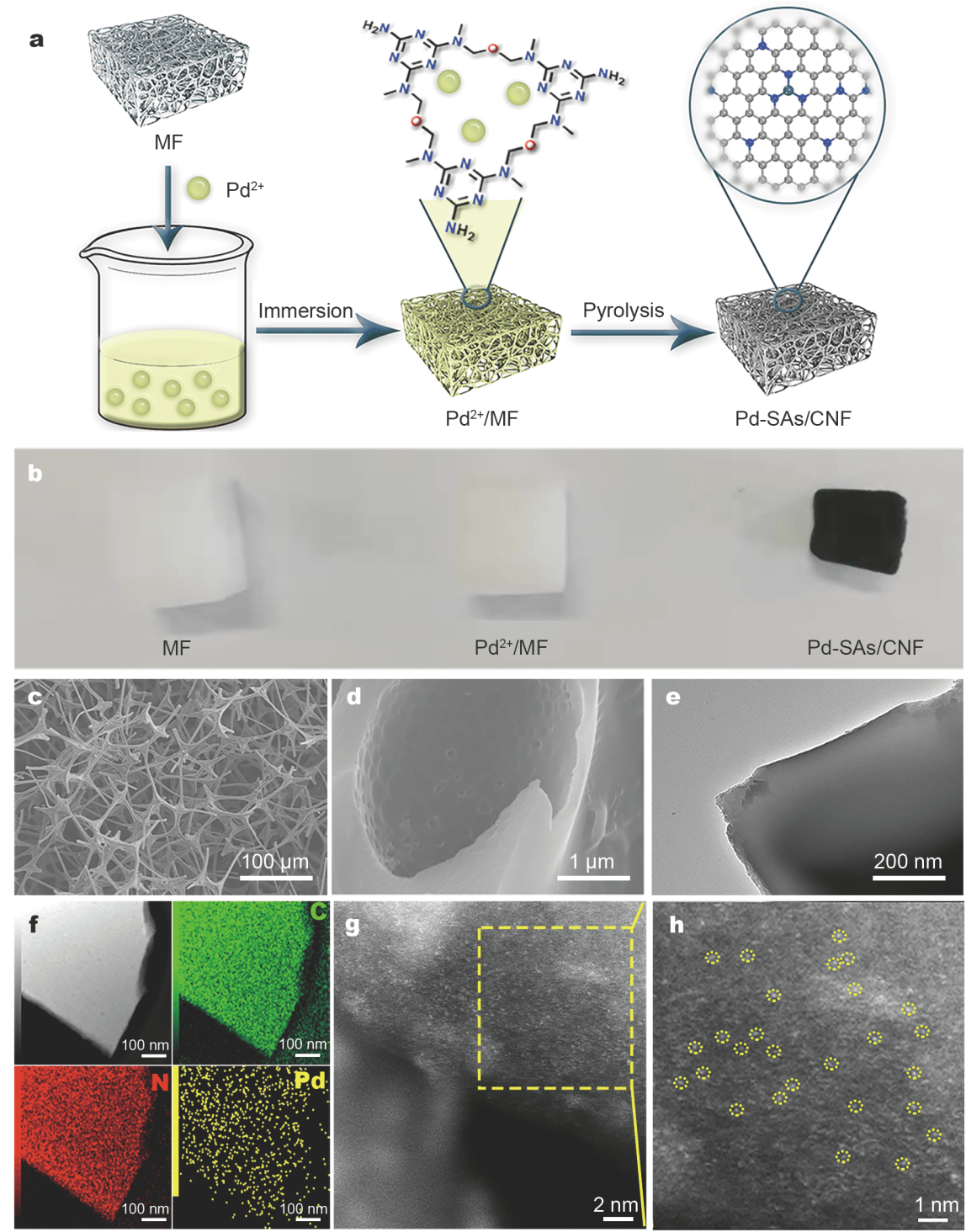

Figure 1 (a) Schematic synthetic process of Pd-SAs/CNF. (b) Photographs of MF, $\mathrm{Pd}^{2+} / \mathrm{MF}$ and monolithic Pd-SAs/CNF catalyst, respectively. (c) SEM, (d) enlarged SEM and (e) TEM images of Pd-SAs/CNF. (f) HAADF-STEM image and EDS maps of Pd-SAs/CNF (C: green; N: red; Pd: yellow). (g, h) AC HAADF-STEM and enlarged images of Pd-SAs/CNF. 
tailored into different macro shapes to meet the needs in different actual operation environments (Fig. $1 \mathrm{~b}$ and Fig. S1).

SEM and enlarged SEM images demonstrate that PdSAs/CNF possesses abundant hierarchically porous structure (Fig. 1c, d). No obvious Pd NPs are observed in the TEM image of Pd-SAs/CNF (Fig. 1e). The XRD pattern of Pd-SAs/CNF exhibits the broad peaks assigned to amorphous carbon (Fig. S2). EDS maps show the homogeneous distribution of Pd (yellow), N (red) and C (green) elements in Pd-SAs/CNF (Fig. 1f). To investigate the chemical state of elements, XPS was carried out. The C 1s spectrum reveals the coexistence of four peaks of $C$ species at the binding energies of $284.5 \mathrm{eV}$ for graphite $\mathrm{C}$, $285.2 \mathrm{eV}$ for $-\mathrm{C}-\mathrm{N}-$ bonds, $286.4 \mathrm{eV}$ for $-\mathrm{C}-\mathrm{OH}$ bonds and $289.2 \mathrm{eV}$ for $-\mathrm{C}=\mathrm{O}-$ bonds (Fig. S3a). The $\mathrm{N} 1 \mathrm{~s}$ spectrum can be deconvoluted into four peaks indexed to $398.1 \mathrm{eV}$ for pyridinic $\mathrm{N}, 399.5 \mathrm{eV}$ for pyrrolic $\mathrm{N}$, $400.9 \mathrm{eV}$ for graphitic $\mathrm{N}$ and $401.9 \mathrm{eV}$ for oxidized $\mathrm{N}$ (Fig. S3b).

To directly observe the dispersion of Pd species at the atomic scale, we conducted the aberration-corrected HAADF-STEM (AC HAADF-STEM) measurements. As shown in Fig. 1g, h, isolated bright dots marked by yellow circles are identified and associated with single Pd atoms, which indicates the atomic dispersion of Pd species in PdSAs/CNF. The hierarchically porous structure of CNF plays a crucial role on the synthesis of Pd-SAs/CNF, as revealed by the presence of obvious Pd NPs dispersed on the surface of nitrogen-doped carbon in the control sample by using powdered melamine-formaldehyde polymer (Figs S4 and S5).

To study the local structure of Pd-SAs/CNF, X-ray absorption near-edge structure (XANES) and extended XAFS (EXAFS) measurements were performed. The XANES data of Pd-SAs/CNF locates between those of Pd foil and PdO (Fig. 2a). And, the average oxidation state of single $\mathrm{Pd}$ atoms in Pd-SAs/CNF is close to +1 (Fig. 2b). The corresponding EXAFS data were handled with $k^{2}$ iteration of Fourier transform (FT). As shown in Fig. 2c, the FT EXAFS curve of Pd K-edge of Pd-SAs/CNF exhibits only one dominant peak at around $1.4 \AA$, which is assigned to the $\mathrm{Pd}-\mathrm{N}$ coordination. Compared with $\mathrm{Pd}$ foil, the peak of Pd-Pd at around $2.4 \AA$ is not detected in $\mathrm{Pd}-\mathrm{SAs} / \mathrm{CNF}$, which indicates the sole presence of isolated Pd atoms in Pd-SAs/CNF.

Wavelet transform (WT) analysis is a powerful tool to identify the atomic structure through discriminating the backscattering atoms and providing both radial distance resolution and $k$-space resolution. To identify the atomic structure, WT analysis was performed. As shown in Fig. 2 d, only one intensity maximum at about $5.0 \AA^{-1}$ from $\mathrm{Pd}-\mathrm{N}$ contribution is observed in the WT contour plots of Pd-SAs/CNF. Compared with the WT contour plots of Pd foil and PdO (Fig. 2d), no intensity maximum near $9.0 \AA^{-1}$ attributed to Pd-Pd path is detected. The combination of AC HAADF-STEM, EXAFS and WT results reveals that $\mathrm{Pd}$ species exist as single atoms in $\mathrm{Pd}$ SAs/CNF. Quantitative EXAFS fitting of Pd-SAs/CNF was carried out and the corresponding fitting parameters are summarized in Fig. 2e, Fig. S6 and Table S1. The bestfitting result suggests that the first shell peak at $1.4 \AA$ is ascribed to isolated $\mathrm{Pd}$ atoms coordinated by three $\mathrm{N}$ atoms as Pd-N3 structure. Based on the above-mentioned structural analysis, DFT calculations optimized and established the structural model of Pd-SAs/CNF (inset of Fig. 2e).

Monolithic Pd-SAs/CNF catalyst possesses outstanding structural strength so it can be readily put into and taken out from the reaction system by tweezer. The products can be easily washed off by fluid washing solvent (Fig. 3a). We selected the selective hydrogenation of NPA to evaluate the catalytic performance of Pd-SAs/CNF. Because both the nitro and alkynyl groups of the NPA substrate are easily reduced, two different catalytic pathways are presented in Fig. 3b. To our surprise, the Pd-SAs/CNF not only exhibits complete transformation of 4nitrophenylethyne after $2 \mathrm{~h}$, but also attains $~ 99 \%$ selectivity of 4-nitrostyrene, which is the selective semihydrogenation product (Fig. 3c). When we extend the reaction time to $5 \mathrm{~h}$, products of excessive hydrogenation (4-ethylnitrobenzene, 4-ethenylaniline or 4-ethylaniline) are almost not detected, indicating the excellent chemical selectivity of Pd-SAs/CNF (Fig. 3d). As shown in the insets of Fig. 3d, monolithic Pd-SAs/CNF catalysts can keep unbroke during the whole catalysis process. To confirm the role of single-atom Pd species, we investigated the catalytic performance of the pure $\mathrm{CNF}$ and no reaction activity was detected, which implies that the single Pd atoms are the active sites. For comparison, the Pd NPs supported on CNF sample, marked as Pd-NPs/ $\mathrm{CNF}$, were synthesized and characterized (Fig. S7). Meanwhile, homogeneous $\mathrm{Pd}(\mathrm{acac})_{2}$ and commercial $\mathrm{Pd} /$ $\mathrm{C}$ catalysts were also employed. All catalytic results are summarized in Table S2 and the selectivities of the hydrogenation reaction towards all Pd catalysts are presented in Fig. 3c. Both the nitro and alkynyl groups of NPA are reduced and almost completely converted into corresponding 4-ethylaniline over the Pd-NPs/CNF and homogeneous $\mathrm{Pd}(\mathrm{acac})_{2}$, indicating that both the Pd-NPs/ 

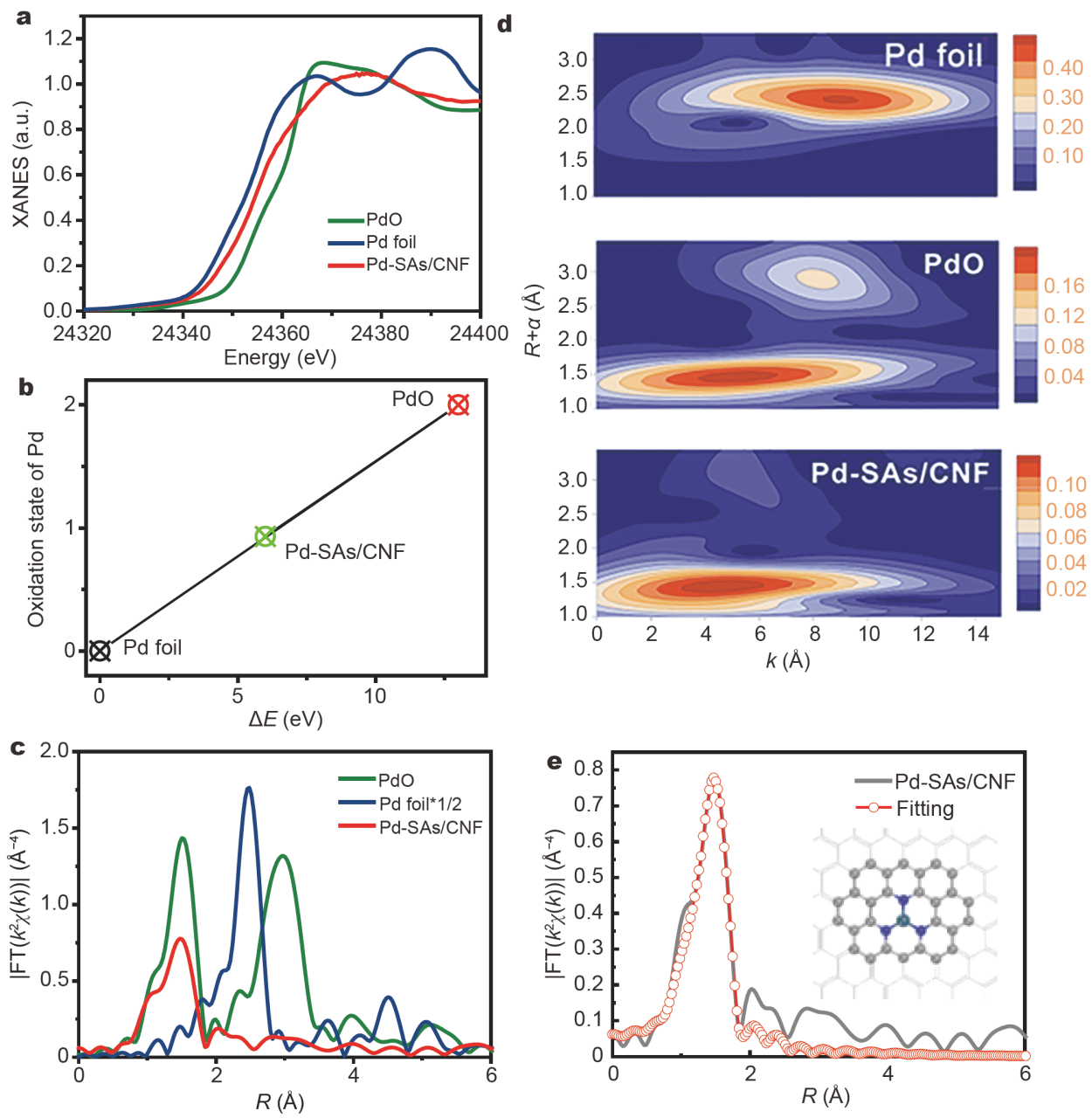

Figure 2 (a) XANES spectra, (b) oxidation state analysis from XANES data, (c) FT-EXAFS spectra of Pd-SAs/CNF, Pd foil and PdO. (d) WT contour plots of Pd foil, PdO and Pd-SAs/CNF. (e) EXAFS fitting curve of Pd-SAs/CNF (inset: optimized model).

$\mathrm{CNF}$ and homogeneous $\mathrm{Pd}(\mathrm{acac})_{2}$ are no selectivity for hydrogenation reaction of NPA. And, the commercial Pd/ C obtains a mixture of products containing $25 \%$ of 4 ethenylaniline and $75 \%$ of 4 -ethylaniline. Based on the above results, we speculate that the extremely high selectivity of Pd-SAs/CNF to 4-nitrostyrene is attributed to isolated site effect induced by single-atom Pd sites, which leads to preferential adsorption of alkynyl group rather than the nitro group (Fig. 3f). Meanwhile, such effect may suppress further hydrogenation of alkenyl group due to limited coordination space.

In catalytic cyclic tests, the Pd-SAs/CNF shows no significant decline in activity and selectivity after five times successive cyclic tests, indicating the good reusability (Fig. 3e). Furthermore, we carefully characterized Pd-SAs/CNF after reusability tests. And the morphology and atomic dispersion of Pd species in the used Pd-SAs/
CNF still maintain (Figs S8-S11), suggesting the excellent stability. To demonstrate the advantages of monolithic $\mathrm{Pd}-\mathrm{SAs} / \mathrm{CNF}$, we designed and synthesized single $\mathrm{Pd}$ atoms supported nitrogen-doped carbon powder, marked as the powdered Pd-SAs/NC (Figs S12 and S13). As shown in Table S2, the powdered Pd-SAs/NC exhibits $38 \%$ conversion ( $89 \%$ selectivity) for the hydrogenation reaction of NPA, which is much lower than that of PdSAs/CNF. In addition, the powdered Pd-SAs/NC shows obvious decline in activity and selectivity after cyclic tests (Fig. S14). These results suggest that monolithic structure of Pd-SAs/CNF enhances catalytic activity and stability and facilitates the separation.

Furthermore, we tested the alkyne scope of selective hydrogenation reactions with Pd-SAs/CNF (Table S3). Phenylacetylene (2a) was well catalyzed in both $\sim 99 \%$ of conversion and selectivity. The substituted phenyl- 
a

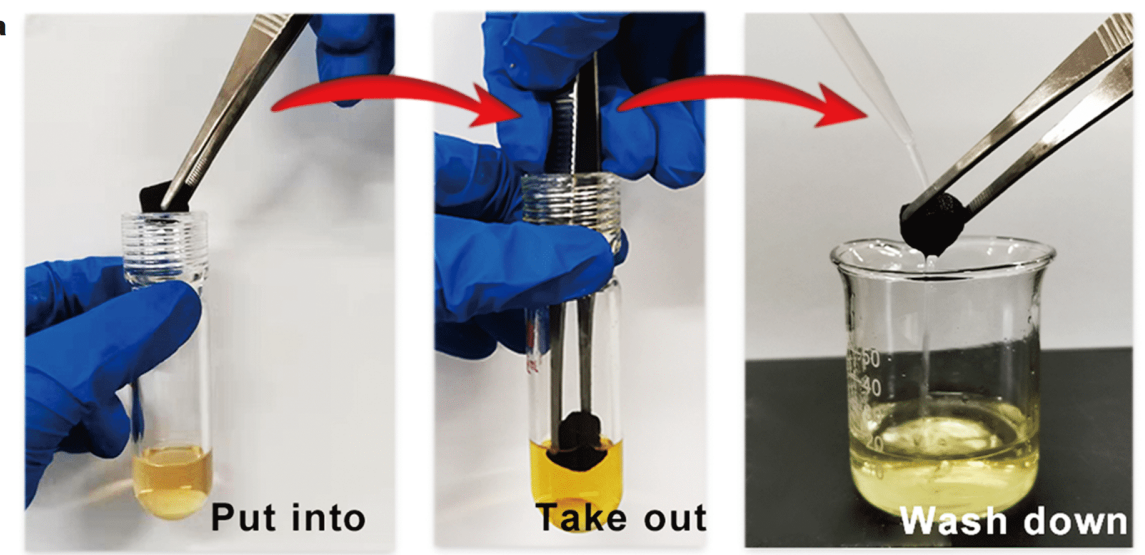

b
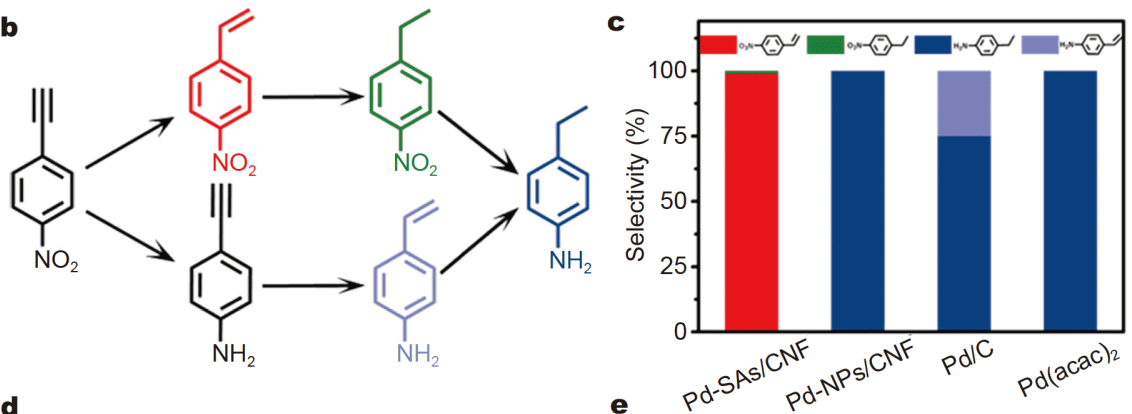

d

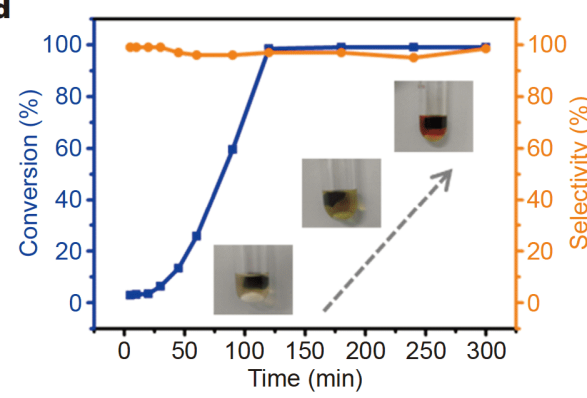

e
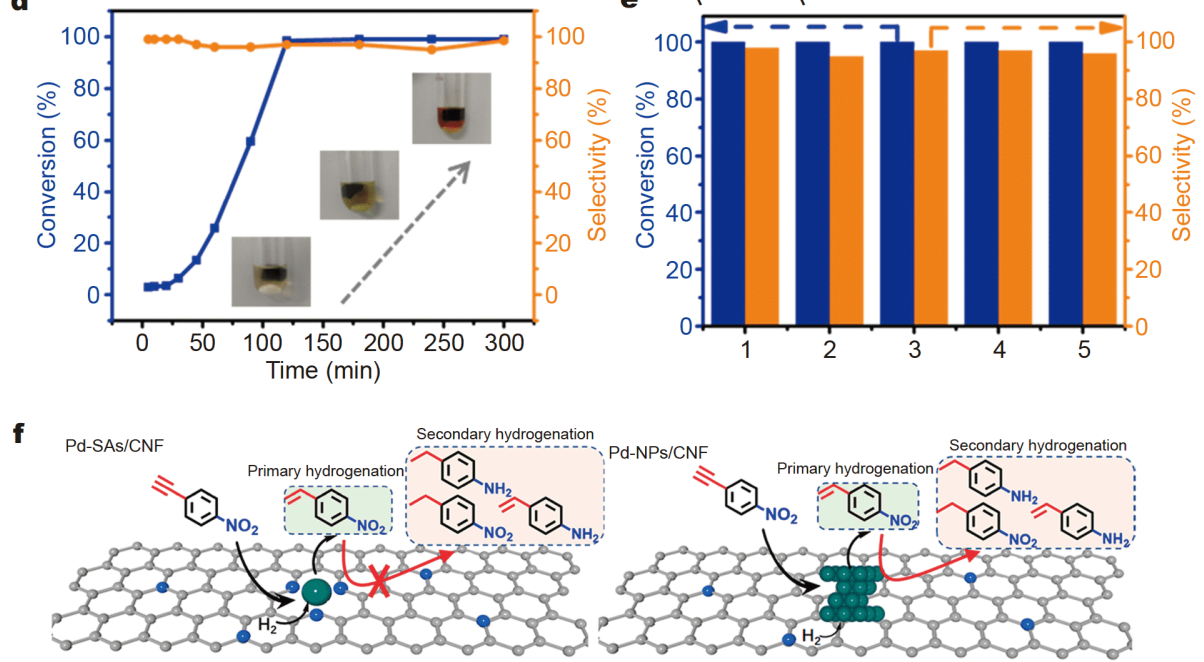

Figure 3 (a) Photograph of monolithic Pd-SAs/CNF catalyst during the catalytic reaction. (b) Possible reaction pathway of 4-nitrophenylacetylene hydrogenation reaction. (c) The selectivity of products for Pd-SAs/CNF, Pd-NPs/CNF, commercial Pd/C and Pd(acac) 2 after 3 h. (d) Conversion and selectivity changes with different reaction times. Insets in (d) show the color change with time. (e) Schematic illustration of enhancement on selectivity of Pd-SAs/CNF compared with Pd-NPs/CNF. (f) Conversion and selectivity of Pd-SAs/CNF during five cycles.

acetylene with both electron-donating groups $(\mathbf{2 b}, \mathbf{2 c}, \mathbf{2 d})$ were reacted smoothly in above $90 \%$ of conversion and above $97 \%$ of selectivity. Halogen-substituted phenylacetylene (2e, 2f) was also catalyzed to give the desired semihydrogenated products in $\sim 99 \%$ of conversions and selectivities. The 2 -ethynylnaphthalene $(\mathbf{2 g})$ with a big-sized naphthyl group achieved excellent conversion and se- lectivity. In addition, the terminal aliphatic alkyne was reacted to give the corresponding product $(\mathbf{2 h})$ in good conversion and selectivity. $N$-heterocyclic alkyne was also converted into corresponding alkene (2i) in $\sim 99 \%$ of conversion and $\sim 99 \%$ of selectivity.

To elucidate the superior selectivity of Pd-SAs/CNF on NPA semi-hydrogenation, DFT calculations were per- 
formed. As shown in Fig. 4a and Fig. S15a, the threecoordinated single-atom Pd (Pd-SA) is protruded out from the graphene, and displays a spin moment of $0.71 \mu_{B}$ and a Bader charge of $+0.57|\mathrm{e}|$, illustrating that it can be essentially assigned as $\mathrm{Pd}^{+}$cation, which accords with the XANES result of Pd-SAs/CNF.

Firstly, the adsorption configurations of NPA on PdSAs show that NPA prefers anchoring on Pd-SAs via the alkynyl (Fig. 4b) to the nitro group (Fig. S15b) [47] with an adsorption free energy $\left(G_{\text {ad }}\right)$ of $-0.72 \mathrm{eV} v s .-0.25 \mathrm{eV}$. Thus, the alkynyl group would in principle serve as the reactive center for hydrogenation. Secondly, the calculation results show that the alkynyl hydrogenation prefers proceeding via path I to path II (in path I, the atomic $\mathrm{H}$ attacks the $\alpha-C$ and then $\beta-C$ of alkynyl group in turn, while this hydrogenation order is inverse for path II,
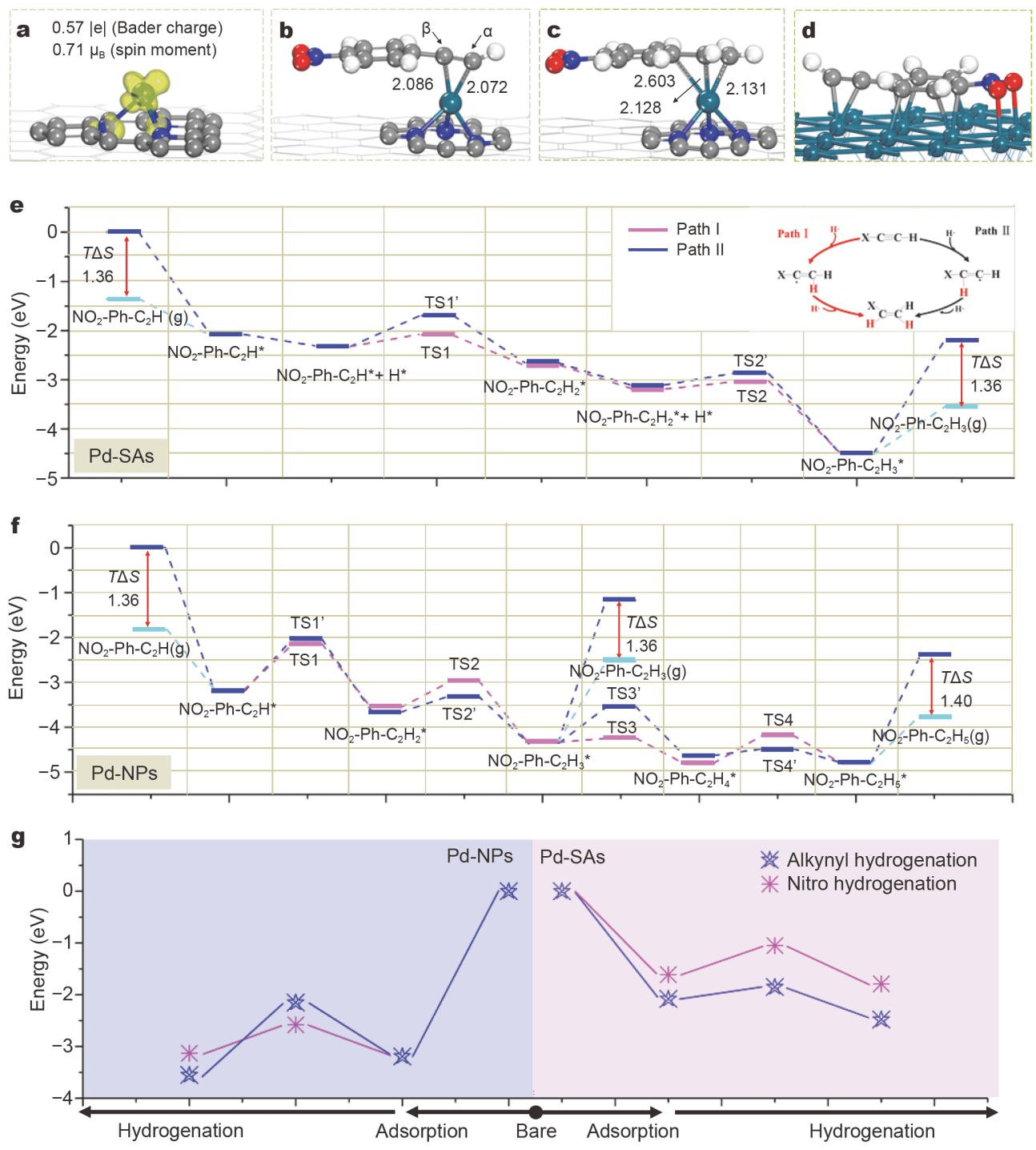

Figure 4 (a) Configurations of Pd-SAs anchored at 3N-doped graphene, with the charge and spin density illustrated (isovalue 0.001 e/bohr ${ }^{3}$ ). (b, c) Geometries (side view) of NPA and 4-nitrostyrene adsorption on Pd-SAs, and (d) NPA adsorption on Pd-NPs. The gray, blue, green, red, and white balls represent $\mathrm{C}, \mathrm{N}, \mathrm{Pd}, \mathrm{O}$, and $\mathrm{H}$ atoms, respectively. (e, f) Energy profiles of alkynyl semi-hydrogenation of NPA into alkenyl on Pd-SAs/CNF, and full hydrogenation into alkyl group on Pd (111), respectively. Note that the free energy profiles were also estimated by correcting the adsorption/ desorption energies (see cyan line), considering the large entropy contributions of gaseous molecules (TAS) at $323 \mathrm{~K}$. Also, the inset in (e) shows two examined hydrogenation pathways, in which path I (red) indicates that alkynyl hydrogenation occurs at $\alpha-C$ initially and then at $\beta$-C, while path II (black) denotes the inverse hydrogenation order; similar denotation was applied on alkenyl hydrogenation on Pd (111). (g) Energetics comparisons of adsorption and initial hydrogenation of NPA on Pd-NPs/CNF and Pd-SAs/CNF. 
Fig. $4 \mathrm{~b}, \mathrm{e})$. The energy profiles of path I in Fig. 4e show that the $\alpha-C$ prefers to be hydrogenated with a low barrier of $0.23 \mathrm{eV}$, and then the vinyl can couple with another $\mathrm{H}$ to form 4-nitrostyrene $\left(\mathrm{NO}_{2}-\mathrm{Ph}-\mathrm{C}_{2} \mathrm{H}_{3}{ }^{*}\right)$ with a barrier of only $0.16 \mathrm{eV}$ (Fig. S15c, d). In other word, the Pd singleatom site has a high activity toward the semi-hydrogenation of NPA into $\mathrm{NO}_{2}-\mathrm{Ph}-\mathrm{C}_{2} \mathrm{H}_{3}{ }^{*}$ through path $\mathrm{I}$. Note that the atomic $\mathrm{H}$ is adopted as hydrogen source considering the widely acknowledged fact that ammonia borane can easily decompose and release $\mathrm{H}[48,49]$.

Interestingly, when further identifying the $\mathrm{NO}_{2}-\mathrm{Ph}$ $\mathrm{C}_{2} \mathrm{H}_{3}{ }^{*}$ adsorption on Pd-SAs (Fig. 4c), $\mathrm{NO}_{2}-\mathrm{Ph}-\mathrm{C}_{2} \mathrm{H}_{3}{ }^{*}$ is found to interact closely with Pd-SAs via the delocalized $\pi$ bond of alkenyl/phenyl group, forming three evident $\mathrm{Pd}-\mathrm{C}$ bonds, which leads to a result that no extra space is left for hydrogen adsorption, evidenced by the hydrogen adsorption energy of only $-0.08 \mathrm{eV}$ in Table S4. Therefore, further hydrogenation of $\mathrm{NO}_{2}-\mathrm{Ph}-\mathrm{C}_{2} \mathrm{H}_{3}{ }^{*}$ will have to cease, and $\mathrm{NO}_{2}-\mathrm{Ph}-\mathrm{C}_{2} \mathrm{H}_{3}{ }^{*}$ will exclusively desorb from Pd-SAs with a desorption free energy of $0.94 \mathrm{eV}$, rationalizing the high semi-hydrogenation selectivity of NPA on Pd-SAs/CNF.

Different from the site-specific adsorption of NPA on Pd-SAs, NPA prefers adsorbing parallelly on the multiatom Pd (111) surface (the most exposed facet of Pd-NPs) using the nitro-, alkynyl- and phenyl-groups collectively (Fig. 4d) [50], with a more negative $G_{\text {ad }}$ of $-1.83 \mathrm{eV}$ (versus $-0.72 \mathrm{eV}$ on Pd-SAs). As detailed calculation results are shown in the Supplementary information (including the energy profiles of the partial/full hydrogenation progress of alkynyl or the hydrogenation/ deoxygenation of nitro $\left(-\mathrm{NO}_{2}\right)$ into $-\mathrm{NH}_{2}$ on Pd-NPs, Fig. S15e-h, Fig. 4 f and Fig. S16, Scheme S1, Table S5), it is found that this unselective chemisorption effect could essentially lead to an inferior selectivity of NPA hydrogenation on Pd-NPs/CNF in comparison with Pd-SAs/ $\mathrm{CNF}$, explaining the experimental result that considerable $\mathrm{NH}_{2}-\mathrm{Ph}-\mathrm{C}_{2} \mathrm{H}_{5}$ were produced on Pd-NPs/CNF.

Overall, in light of the above results, the superior selectivity of NPA semi-hydrogenation into 4-nitrostyrene on Pd-SAs/CNF compared with Pd-NPs/CNF could be ascribed to two key factors: (i) the three-coordinated Pd single-atom site in Pd-SAs/CNF can preferentially adsorb alkynyl group rather than the nitro group, which is different from that on Pd-NPs/CNF (Fig. 4g); (ii) the limited coordination space of isolated Pd single-atom site, which could be the prominent structural feature of SAC, inhibits the further hydrogenation of alkenyl group. These DFT calculation analyses coincide with experimental results, suggesting the isolated site effect of single atoms for en- hancement on catalytic selectivity.

\section{CONCLUSIONS}

In summary, we have developed a practical strategy to synthesize monolithic Pd SACs. Moreover, we demonstrate an isolated site effect of Pd-SAs/CNF to remarkably improve the selectivity in NPA semi-hydrogenation compared with conventional heterogeneous Pd nanocatalysts and homogeneous Pd complex catalysts. In addition, the monolithic structure of Pd-SAs/CNF favors separation and subsequent recycling, which also leads to excellent reusability and stability. Notably, monolithic nature of the Pd-SAs/CNF catalyst makes it easy to separate from the reaction system, which is beneficial for conducting the subsequent recycling. We believe the discovery of isolated site effect and the development of monolithic SACs may open up new opportunities to design highly selective and practical catalysts for selective catalysis.

Received 20 October 2020; accepted 25 November 2020; published online 9 February 2021

1 Kyriakou G, Boucher MB, Jewell AD, et al. Isolated metal atom geometries as a strategy for selective heterogeneous hydrogenations. Science, 2012, 335: 1209-1212

2 Macino M, Barnes AJ, Althahban SM, et al. Tuning of catalytic sites in $\mathrm{Pt} / \mathrm{TiO}_{2}$ catalysts for the chemoselective hydrogenation of 3-nitrostyrene. Nat Catal, 2019, 2: 873-881

3 Huang $\mathrm{C}$, Huang $\mathrm{Y}$, Liu C, et al. Integrating hydrogen production with aqueous selective semi-dehydrogenation of tetrahydroisoquinolines over a $\mathrm{Ni}_{2} \mathrm{P}$ bifunctional electrode. Angew Chem Int Ed, 2019, 58: 12014-12017

4 Wu Y, Liu C, Wang C, et al. Selective transfer semihydrogenation of alkynes with $\mathrm{H}_{2} \mathrm{O}\left(\mathrm{D}_{2} \mathrm{O}\right)$ as the $\mathrm{H}(\mathrm{D})$ source over a Pd-P cathode. Angew Chem Int Ed, 2020, 132: 21356-21361

5 Zhou S, Shang L, Zhao Y, et al. Pd singleatom catalysts on nitrogen-doped graphene for the highly selective photothermal hydrogenation of acetylene to ethylene. Adv Mater, 2019, 31: 1900509

6 Fu S, Chen NY, Liu X, et al. Ligand-controlled cobalt-catalyzed transfer hydrogenation of alkynes: Stereodivergent synthesis of $Z$ and E- alkenes. J Am Chem Soc, 2016, 138: 8588-8594

7 Blaser HU, Malan C, Pugin B, et al. Selective hydrogenation for fine chemicals: Recent trends and new developments. Adv Synthesis Catal, 2003, 345: 103-151

8 Chen Y, Gao R, Ji S, et al. Atomic-level modulation of electronic density at cobalt single-atom sites derived from metal-organic frameworks: Enhanced oxygen reduction performance. Angew Chem Int Ed, 2021, 60: 3212-3221

9 Lin R, Albani D, Fako E, et al. Design of single gold atoms on nitrogen-doped carbon for molecular recognition in alkyne semihydrogenation. Angew Chem Int Ed, 2019, 58: 504-509

10 Sun $\mathrm{T}, \mathrm{Xu} \mathrm{L}$, Wang $\mathrm{D}$, et al. Metal organic frameworks derived single atom catalysts for electrocatalytic energy conversion. Nano Res, 2019, 12: 2067-2080

11 Ji S, Qu Y, Wang T, et al. Rare-earth single erbium atoms for 
enhanced photocatalytic $\mathrm{CO}_{2}$ reduction. Angew Chem Int Ed, 2020, 59: 10651-10657

12 Zhang J, Zheng C, Zhang M, et al. Controlling N-doping type in carbon to boost single-atom site $\mathrm{Cu}$ catalyzed transfer hydrogenation of quinoline. Nano Res, 2020, 13: 3082-3087

13 Mao J, He CT, Pei J, et al. Isolated $\mathrm{Ni}$ atoms dispersed on $\mathrm{Ru}$ nanosheets: High-performance electrocatalysts toward hydrogen oxidation reaction. Nano Lett, 2020, 20: 3442-3448

14 Song J, Liu S, Yang C, et al. The role of $\mathrm{Al}$ doping in $\mathrm{Pd} / \mathrm{ZnO}$ catalyst for $\mathrm{CO}_{2}$ hydrogenation to methanol. Appl Catal B-Environ, 2020, 263: 118367

15 Yang J, Li W, Wang D, et al. Single-atom materials: Small structures determine macroproperties. Small Struct, 2020, 2000051

16 Cui X, Li W, Ryabchuk P, et al. Bridging homogeneous and heterogeneous catalysis by heterogeneous single-metal-site catalysts. Nat Catal, 2018, 1: 385-397

17 Yang J, Li W, Wang D, et al. Electronic metal-support interaction of single-atom catalysts and applications in electrocatalysis. Adv Mater, 2020, 32: 2003300

18 Zhang N, Ye C, Yan H, et al. Single-atom site catalysts for environmental catalysis. Nano Res, 2020, 13: 3165-3182

19 Li X, Rong H, Zhang J, et al. Modulating the local coordination environment of single-atom catalysts for enhanced catalytic performance. Nano Res, 2020, 13: 1842-1855

20 Lin L, Zhou W, Gao R, et al. Low-temperature hydrogen production from water and methanol using $\mathrm{Pt} / \mathrm{a}-\mathrm{MoC}$ catalysts. Nature, 2017, 544: 80-83

$21 \mathrm{Xu} \mathrm{Q}$, Guo CX, Tian S, et al. Coordination structure dominated performance of single-atomic Pt catalyst for anti-Markovnikov hydroboration of alkenes. Sci China Mater, 2020, 63: 972-981

22 Tian $\mathrm{S}, \mathrm{Hu} \mathrm{M}, \mathrm{Xu} \mathrm{Q}$, et al. Single-atom $\mathrm{Fe}$ with $\mathrm{Fe}_{1} \mathrm{~N}_{3}$ structure showing superior performances for both hydrogenation and transfer hydrogenation of nitrobenzene. Sci China Mater, 2021, 64: $642-650$

23 Lin Y, Liu P, Velasco E, et al. Fabricating single-atom catalysts from chelating metal in open frameworks. Adv Mater, 2019, 31: 1808193

24 Fei H, Dong J, Chen D, et al. Single atom electrocatalysts supported on graphene or graphene-like carbons. Chem Soc Rev, 2019, 48: 5207-5241

25 Guo X, Fang G, Li G, et al. Direct, nonoxidative conversion of methane to ethylene, aromatics, and hydrogen. Science, 2014, 344: 616-619

26 Malta G, Kondrat SA, Freakley SJ, et al. Identification of single-site gold catalysis in acetylene hydrochlorination. Science, 2017, 355: 1399-1403

27 Jiao L, Zhang R, Wan G, et al. Nanocasting $\mathrm{SiO}_{2}$ into metal-organic frameworks imparts dual protection to high-loading Fe singleatom electrocatalysts. Nat Commun, 2020, 11: 2831

28 Wang L, Zhang W, Wang S, et al. Atomic-level insights in optimizing reaction paths for hydroformylation reaction over $\mathrm{Rh} / \mathrm{CoO}$ single-atom catalyst. Nat Commun, 2016, 7: 14036

29 Zhao R, Liang Z, Gao S, et al. Puffing up energetic metal-organic frameworks to large carbon networks with hierarchical porosity and atomically dispersed metal sites. Angew Chem Int Ed, 2019, 58: $1975-1979$

30 Su H, Gao P, Wang MY, et al. Grouping effect of single nickel- $\mathrm{N}_{4}$ sites in nitrogen-doped carbon boosts hydrogen transfer coupling of alcohols and amines. Angew Chem Int Ed, 2018, 57: 1519415198
31 Yang S, Kim J, Tak YJ, et al. Single-atom catalyst of platinum supported on titanium nitride for selective electrochemical reactions. Angew Chem Int Ed, 2016, 55: 2058-2062

32 Hülsey MJ, Zhang J, Yan N. Harnessing the wisdom in colloidal chemistry to make stable single-atom catalysts. Adv Mater, 2018, 30: 1802304

33 Zhuang Z, Kang Q, Wang D, et al. Single-atom catalysis enables long-life, high-energy lithium-sulfur batteries. Nano Res, 2020, 13: 1856-1866

34 Xu Y, Zhang Z, Geng X, et al. Smart carbon foams with switchable wettability for fast oil recovery. Carbon, 2019, 149: 242-247

35 Sun T, Li Y, Cui T, et al. Engineering of coordination environment and multiscale structure in single-site copper catalyst for superior electrocatalytic oxygen reduction. Nano Lett, 2020, 20: 62066214

$36 \mathrm{Xu} \mathrm{B}$, Zhang Z, Wang X. Formamide: An efficient solvent to synthesize water-soluble and sub-ten-nanometer nanocrystals. Nanoscale, 2013, 5: 4495-4505

37 Mou S, Lu Y, Jiang Y. A facile and cheap coating method to prepare $\mathrm{SiO}_{2}$ /melamine-formaldehyde and $\mathrm{SiO}_{2}$ /urea-formaldehyde composite microspheres. Appl Surf Sci, 2016, 384: 258-262

38 Perdew JP, Burke K, Ernzerhof M. Generalized gradient approximation made simple. Phys Rev Lett, 1996, 77: 3865-3868

39 Kresse G, Furthmüller J. Efficiency of ab-initio total energy calculations for metals and semiconductors using a plane-wave basis set. Comput Mater Sci, 1996, 6: 15-50

40 Kresse G, Joubert D. From ultrasoft pseudopotentials to the projector augmented-wave method. Phys Rev B, 1999, 59: 1758-1775

41 Kresse G, Furthmüller J. Efficient iterative schemes for ab initio total-energy calculations using a plane-wave basis set. Phys Rev B, 1996, 54: 11169-11186

42 Blöchl PE, Jepsen O, Andersen OK. Improved tetrahedron method for Brillouin-zone integrations. Phys Rev B, 1994, 49: 16223-16233

43 Grimme S, Antony J, Ehrlich S, et al. A consistent and accurate $a b$ initio parametrization of density functional dispersion correction (DFT-D) for the 94 elements H-Pu. J Chem Phys, 2010, 132: 154104

44 Wang HF, Wang D, Liu X, et al. Unexpected C-C bond cleavage mechanism in ethylene combustion at low temperature: Origin and implications. ACS Catal, 2016, 6: 5393-5398

45 Wang HF, Gong XQ, Guo YL, et al. A model to understand the oxygen vacancy formation in $\mathrm{Zr}$-Doped $\mathrm{CeO}_{2}$ : Electrostatic interaction and structural relaxation. J Phys Chem C, 2009, 113: 1022910232

46 Jin J, Sun $\mathrm{N}, \mathrm{Hu} \mathrm{W}$, et al. Insight into room-temperature catalytic oxidation of nitric oxide by $\mathrm{Cr}_{2} \mathrm{O}_{3}$ : A DFT study. ACS Catal, 2018, 8: $5415-5424$

47 Kim SK, Han WS, Kim TJ, et al. Palladium catalysts for dehydrogenation of ammonia borane with preferential B-H activation. J Am Chem Soc, 2010, 132: 9954-9955

48 Ai D, Guo Y, Liu W, et al. DFT studies on catalytic dehydrogenation of ammonia borane by $\mathrm{Ni}(\mathrm{NHC})_{2}$. J Phys Org Chem, 2014, 27: 597-603

49 Ma LL, Lv CQ, Wang GC. A DFT study and micro-kinetic analysis of acetylene selective hydrogenation on Pd-doped $\mathrm{Cu}(111)$ surfaces. Appl Surf Sci, 2017, 410: 154-165

50 Mahata A, Rai RK, Choudhuri I, et al. Direct vs. indirect pathway for nitrobenzene reduction reaction on a $\mathrm{Ni}$ catalyst surface: A density functional study. Phys Chem Chem Phys, 2014, 16: 2636526374 
Acknowledgements This work was supported by the National Key R\&D Program of China (2018YFA0702003), the National Natural Science Foundation of China (21890383, 21671117, 21871159 and 21901135), the National Postdoctoral Program for Innovative Talents (BX20180160), and the China Postdoctoral Science Foundation (2018M640113). We thank the BL14W1 station in Shanghai Synchrotron Radiation Facility (SSRF) and 1W1B station for XAFS measurement in Beijing Synchrotron Radiation Facility (BSRF).

Author contributions Li Y, Wang D and Chen Y conceived the idea, designed the research project and wrote the manuscript; Zhang $\mathrm{Z}$ designed the synthesis and controlled experiments, collected and analyzed the data, and wrote the manuscript; Zhou M and Wang $\mathrm{H}$ contributed to the computational results and wrote the manuscript; Liu S helped to perform XAFS data analysis; Zhang J helped with catalytic experiments; Ji S contributed to characterizations of samples and data analysis. All the authors commented on the manuscript and have given approval to the final version of the manuscript.

Conflict of interest The authors declare that they have no conflict of interest.

Supplementary information Experimental details and supporting data are available in the online version of the paper.

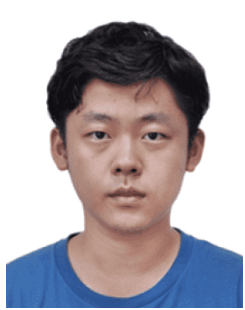

Zedong Zhang received his BSc degree from the College of Chemical Engineering at Beijing University of Chemical Technology in 2019. He is now a $\mathrm{PhD}$ candidate in Prof. Dingsheng Wang's group at the Department of Chemistry, Tsinghua University. His research interests focus on the explorations of the relationships between different configurations and the functions of isolated single-atom-site catalyst.

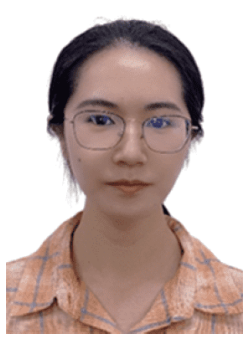

Min Zhou is currently a PhD student under the supervision of Prof. Hai-Feng Wang at the Centre for Computational Chemistry, East China University of Science and Technology (ECUST). Her research interests lie in the first-principle simulations of solid and interface structure, theoretical photo- and electro-catalysis, and firstprinciple simulations of catalytic reactions.

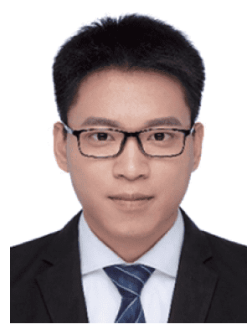

Yuanjun Chen received his BSc degree from the College of Chemical Engineering at Beijing University of Chemical Technology in 2015, and his $\mathrm{PhD}$ degree at the Department of Chemistry, Tsinghua University in 2020 under the supervision of Prof. Yadong Li. His research interests focus on the syntheses and applications of functional nanocatalysts and single-atom site catalysts.

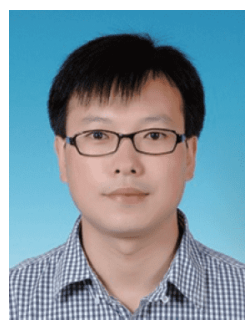

Haifeng Wang received his $\mathrm{PhD}$ degree from ECUST in 2012, and is now a professor at ECUST. His research interests lie in theoretical catalysis and computational chemistry, with the focus on photoelectrocatalysis and rational design of heterogeneous catalyst driven by $\mathrm{AI}$ and $a b$ initio kinetics.

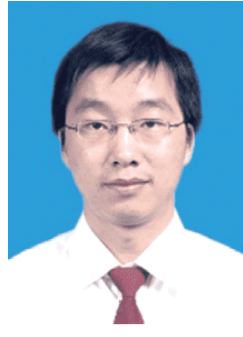

Dingsheng Wang received his BSc degree from the Department of Chemistry and Physics, University of Science and Technology of China in 2004, and his $\mathrm{PhD}$ degree from the Department of Chemistry, Tsinghua University in 2009, under the supervision of Prof. Yadong Li. He did his postdoctoral research at the Department of Physics, Tsinghua University, with Prof. Shoushan Fan. He joined the faculty of the Department of Chemistry, Tsinghua University in 2012.

Pd单原子整体催化剂: 功能化的三维结构和优异 的化学加氢选择性

张泽栋 ${ }^{1 \dagger}$, 周敏 ${ }^{2 \dagger}$, 陈远均 ${ }^{*}$, 柳守杰 ${ }^{3}$, 王海丰 ${ }^{2^{*}}$, 张剑 ${ }^{1}$, 冀淑方 $^{1}$, 王定胜 ${ }^{1 *}$, 李亚栋 ${ }^{1}$

摘要 原子级别调控催化剂在选择性加氢反应中的选择性是一个 巨大的挑战. 在本文中, 我们报告了一种简单实用的策略, 用于合成 $\mathrm{Pd}$ 单原子负载在氮掺杂碳纳米泡沫 $(\mathrm{Pd}-\mathrm{SAs} / \mathrm{CNF})$ 上的整体型单原 子催化剂. 此外, 我们证明独特电子结构的单原子 Pd位点使得 $\mathrm{Pd}$ $\mathrm{SAs} / \mathrm{CNF}$ 产生孤立位点效应, 进而导致在4-硝基苯基乙炔半氢化反 应中具有出色的活性和选择性. 此外, 得益于较高的完整性和良好 的机械强度, 整体型Pd-SAs/CNF催化剂易与反应体系分离, 进一步 有利于回收循环利用. 循环测试表明, 整体型Pd-SAs/CNF催化剂具 有优异的可重复使用性和稳定性. 孤立位点效应的发现为设计高 选择性催化剂提供了一种新方法. 整体式单原子催化剂的研究为 推进单原子催化剂的实际应用提供了新的机会. 